\title{
COMPARATIVO ENTRE O QUESTIONÁRIO PSS - BRASIL E TESTES ESPECÍFICOS DE OMBRO EM PACIENTES COM SÍNDROME DO IMPACTO
}

\author{
Daniela Cristine Stenger*, Dianara Schafer*, Altair Argentino Pereira Júnior** \\ Autor Correspondente: Altair Argentino Pereira Júnior E-mail: junior-alt@hotmail.com \\ * Graduada em Fisioterapia pela Faculdade Metropolitana de Blumenau \\ ** Fisioterapeuta, mestre em Ciências do Movimento Humano pela Universidade do Estado de Santa Catarina, docente do \\ Curso de Fisioterapia da Faculdade Metropolitana de Blumenau
}

\begin{abstract}
Resumo
A Síndrome do Impacto é a patologia mais comum que acomete a cintura escapular. Os movimentos de grande amplitude são os mais afetados juntamente com a sobrecarga e os movimentos repetitivos exercidos sobre o membro. Objetivo: comparar o questionário PSS - Brasil e testes específicos para ombro em pacientes com a síndrome do impacto. Metodologia: Participaram do estudo 15 indivíduos com média de idade de 49,8 anos. A coleta de dados foi obtida através da aplicação do questionário o qual tem a finalidade de analisar a disfunção do ombro em três aspectos: dor, satisfação e função e a aplicação de dois testes específicos: Neer e Hawkins - Kennedy, que são indicativos de positividade para a patologia. Resultados: No aspecto de dor, a dor ao esforço obteve a maior média o que condiz com os testes que obtiveram valores positivos para irritabilidade da articulação do ombro, e em contrapartida a satisfação teve uma pontuação abaixo da média. No aspecto função, as AVD's que causaram maior desconforto, foram as que exigiram maior amplitude de movimento e sobrecarga no membro superior, o que foi comprovado com os testes que são irritativos e exijam sobrecarga para a realização do mesmo. Conclusão: Os resultados permitiram concluir que a associação dos testes com o questionário PSS - Brasil são ferramentas úteis na avaliação fisioterapêutica da Síndrome do Impacto.
\end{abstract}

Palavras-Chave: Ombro; Dor; Movimento; Questionário. 


\title{
COMPARATIVE BETWEEN THE PSS - BRAZIL QUESTIONNAIRE AND SHOULDER SPECIFIC TESTS IN PATIENTS WITH THE IMPACT SYNDROME
}

\begin{abstract}
The impact syndrome is the most common pathology which happens at the scapula waist. The movements of great amplitude are the most affected with the overload and the repetitive movements exercised on the limb. Purpose: The purpose of this study was comparing the questionnaire PSS - Brazil and specific tests for the shoulder in patients with the impact syndrome. Methods: 15 people in average 49,8 years old participated on the study. The data collection was obtained through the questionnaire application which aim is to analyze the shoulder dysfunction in three aspects: pain, satisfaction, function and a two-test application: Neer and Hawkins - Kennedy, which are indicatives of positivity for the pathology. Results: In terms of pain to the effort obtained the highest average which matches the positive tests for irritability in the shoulder articulation, and conflicting the satisfaction had a low average. In terms of function the AVD's that caused most discomfort, were the ones which required more movement amplitude and the limb overload, which was testified with the irritative tests and demand overload to happen. Conclusion: The results permitted to conclude that the tests association with the questionnaire PSS - Brazil are useful tools in the physiotherapy evaluation of the impact syndrome.
\end{abstract}

Keywords: Shoulder; Pain; Movement; Questionnaire.

\section{INTRODUÇÃO}

O ombro é composto pelas articulações glenoumeral, acromioclavicular, esternoclavicular e escapulotorácica e com o auxílio da musculatura que age sobre ele, torna-se a articulação mais dinâmica e móvel do corpo. As quatro articulações associadas aos seus componentes são responsáveis por produzir os movimentos necessários para o funcionamento normal do complexo do ombro. Para haver um movimento harmônico todas as articulações agem em conjunto, pois não aconteceria o mesmo com o trabalho individual das articulações. $\mathrm{O}$ ombro tem a função de ligar o membro superior ao tronco e para uma função eficiente o mesmo age em conjunto com o cotovelo para posicionar a mão no espaço. ${ }^{(1)}$
A cintura escapular é uma estrutura muito vulnerável a lesões devido a vários estresses em que é envolvida, apesar disso, ela é muito eficaz na realização de muitos movimentos. Com sua complexidade é a articulação mais móvel e menos estável do corpo e, para sua estabilidade e posicionamento ela depende das articulações musculoesqueléticas vizinhas. ${ }^{(2)}$

A patologia Síndrome do Impacto do ombro que foi descrita pela primeira vez em 1972 por Charles Neer, tem como característica um espectro contínuo e, ao processo final depois de passado primeiramente por uma inflamação e secundariamente por uma fibrose, acaba com a ruptura do manguito rotador. $\mathrm{O}$ tendão do supra-espinhoso, o tendão 
da cabeça longa do bíceps, a bursa subacromial e, com menos intensidade o tendão do infra-espinhoso são as estruturas que no impacto mais sofrem. (3,4)

Devido a várias passagens em que o manguito rotador executa por baixo do arco coracoacromial é onde síndrome do impacto se acentuada. Sendo o tendão do supra-espinhoso o mais acometido e, um pouco menos o infra-espinhoso, assim como também a hipertrofia da bursa subacromial que pode ficar fibrótica, o que piora e reduz cada vez mais o espaço já em comprometimento. ${ }^{(5-8)}$

A Síndrome do Impacto é a afecção mais comum na cintura escapular e acomete mais indivíduos adultos entre 40 e 50 anos, podendo acometer ambos os lados. Essas afecções se dão por alterações que comprometem a estrutura e função deste complexo articular ocasionadas por algumas atividades de trabalho, principalmente em funções em que o membro superior permanece por longos períodos em elevação. ${ }^{(9)}$

Para a síndrome do impacto, a intervenção fisioterapêutica é a primeira opção de tratamento, pois é essencial para melhora do equilíbrio muscular, diminuição do quadro álgico e com isso o indivíduo retorna mais rapidamente para suas AVD's não prejudicando tanto a sua situação socioeconômica. ${ }^{(10)}$

O questionário The Penn Shoulder Score (PSS) criado em 1999, foi traduzido e adaptado para o português do Brasil com base no protocolo Beaton et al., ${ }^{(1)}$ utilizado pela American Academy of Orthopaedic Surgeons (AAOS). E pela International Quality of Life Assessment (IQOLA), ficou reconhecido como PSS-Brasil. Este questionário tem a finalidade de analisar indivíduos com disfunção no ombro, incluindo: dor, satisfação e função, numa escala de 100 pontos. $^{(12)}$

Foram avaliados pacientes com diagnóstico clínico de Síndrome do Impacto com os seguintes testes: Teste do Impacto de Neer: O avaliador estabiliza a escápula do paciente com a mão e com a outra eleva rapidamente o membro superior para frente causando um "choque" do tubérculo maior contra o bordo ântero-inferior do acrômio. Segundo Magee ${ }^{(13)}$ o teste é indicador de uma lesão por excesso de uso do músculo supra - espinhoso e à vezes do tendão do bíceps, onde o paciente refere dor.

Teste do Impacto Hawkins - Kennedy: O avaliador flexiona para frente o membro superior a $90^{\circ} \mathrm{e}$ a seguir roda medialmente o ombro. Este teste avalia limitação do tendão supra-espinhoso.(14) Este movimento empurra o tendão supra- espinhal de encontro à superfície anterior do ligamento coracoacromial e processo coracóide. A dor indica positividade para tendinite do supra-espinhal. ${ }^{(13)}$

Para tornar um estudo mais específico fez-se a escolha destes dois testes específicos por considerarmos de maior irritabilidade para a Síndrome do Impacto onde estabelece uma relação com o questionário PSS- Brasil que é totalmente direcionado para o ombro, tornando-o assim um estudo mais completo para esta patologia, sendo também um adicional ao tratamento fisioterapêutico.

O objetivo deste estudo foi realizar um comparativo entre testes especiais para ombro e o questionário PSS - Brasil.

\section{METODOLOGIA}

Trata-se de um estudo quantitativo, descritivo e bibliográfico. O estudo foi previamente aprovado pelo Comitê de Ética da Faculdade Metropolitana de Blumenau (FAMEBLU), campus II, protocolo 074/2012 Os dados foram recolhidos pelos pesquisadores na clínica Centro de Reabilitação e Fisioterapia (CERFIS) localizada na cidade de Camboriú e, na clínica Centro Fisioterapêutico Fonte Luminosa (UNIFISIO) localizada na cidade de Blumenau.

Antes da avaliação foi entregue e explicado o Termo de Consentimento Livre Esclarecido (TCLE), e então foi entregue e explicado o questionário The Penn Shoulder Score (PSS) traduzido para PSS Brasil, que se trata de um questionário desenvolvido em 1999 para fazer uma análise em indivíduos com disfunção no ombro, ele é constituído por 
uma escala de 100 pontos que inclui três aspectos: dor, satisfação e função.

Três itens são avaliados para ver os domínios de dor e apenas um item é avaliado para ver o grau de satisfação, esses quatro quesitos são avaliados por uma Escala de Avaliação Numérica (EN) de o a 10, sendo que para os itens de dor o número o corresponde à ausência de dor e 10 corresponde à pior dor possível sendo que a pontuação máxima obtida é de 30 pontos e, para o item satisfação o número o corresponde a não satisfeito e o 10 corresponde a muito satisfeito e a pontuação máxima obtida neste item é de 10 pontos. A avaliação da função contém vinte itens, que são pontuados em uma escala de Likert de quatro pontos, sendo que o, corresponde à "não consigo fazer de forma alguma", e 3, "sem dificuldades", sendo que 60 é a pontuação máxima. A pontuação total do PSS varia de o a 100 pontos sendo que a pontuação máxima indica ausência de dor, alta satisfação e boa função. ${ }^{(12)}$

Além do questionário foram aplicados dois testes para também avaliar a função do ombro, são eles: O teste de Neer o qual avalia o manguito rotador, para detectar uma possível lesão. O teste é aplicado com o paciente sentado, o terapeuta realiza passivamente uma abdução do ombro até $90^{\circ}$, o paciente é orientado para que desça o braço lentamente, e, se até a posição final do ombro o paciente referir dor, o teste é indicativo para lesão do manguito rotador. ${ }^{(15)}$
E o Teste de Hawkins-Kennedy que avalia a presença de impacto no ombro, com o envolvimento do tendão supra-espinhoso. O paciente é orientado a flexionar as articulações do ombro e cotovelo a $90^{\circ}$ e o terapeuta realiza uma rotação interna forçada do ombro, isso faz com que comprima o supra-espinhoso contra a porção anterior do ligamento coracoacromial. Se o paciente referir dor o teste é positivo para impacto do supra-espinhoso. (15)

Foram utilizados como critérios de inclusão, pacientes com diagnóstico médico de Síndrome do Impacto. Como critérios de exclusão, pacientes que passaram por algum processo cirúrgico no ombro e pacientes com comprometimento neurológico e cognitivo.

Os dados foram tabulados e organizados em tabelas e gráficos usando o software Microsoft Excel 2010. Após fez-se uso da estatística descritiva para análise dos dados.

\section{RESULTADOS}

Foram 15 pessoas avaliadas com idade que variava de 30 a 74 anos (média de 49 anos e 8 meses), sendo que 10 eram do gênero feminino $(66,6 \%) \mathrm{e}$ 5 eram do gênero masculino $(33,3 \%)$. 0 membro superior direito foi dominante em 12 indivíduos (80\%) e estava acometido em $47 \%$ dos casos, conforme é demonstrado na Tabela 1.

Tabela 1 - Dados demográficos dos indivíduos com síndrome do impacto, obtidos pela aplicação do questionário PSS Brasil

\begin{tabular}{cccccc}
\hline Pacientes & IDAde & Sexo & Função Acometido & $\begin{array}{c}\text { Lado } \\
\text { Dominante }\end{array}$ & Lado \\
\hline S. A & 30 & F & Téc.Enferm. & E & D \\
G. B & 42 & M & Motorista & E & Ambos \\
P. L & 37 & F & Talh.Amostra & E & E \\
M. R.S & 37 & F & Serviços Gerais & D & D \\
C. R.S & 55 & F & Cozinheira & D & D \\
A. R. B & 63 & F & Do Lar & D & D
\end{tabular}




$\begin{array}{llcccc}\text { P. C. A } & 52 & \text { M } & \text { Autônomo } & \text { E } & \text { D } \\ \text { A. C.M } & 47 & \text { M } & \text { Carpinteiro } & \text { E } & \text { D } \\ \text { C. A.S } & 39 & \text { F } & \text { Serviços Gerais } & \text { E } & \text { D } \\ \text { H. J.S } & 45 & \text { M } & \text { Pedreiro } & \text { D } & \text { D } \\ \text { N. A.S } & 54 & \text { F } & \text { Do Lar } & \text { D } & \text { D } \\ \text { E. V.S.S } & 55 & \text { F } & \text { Do Lar } & \text { E } & \text { D } \\ \text { E. C } & 73 & \text { F } & \text { Do Lar } & \text { D } & \text { D } \\ \text { D. A. A } & 74 & \text { M } & \text { Mil. Reformado } & \text { E } & \text { E } \\ \text { N. J.C } & 44 & \text { F } & \text { Costureira } & \text { D } & \text { D }\end{array}$

Os pacientes avaliados apresentaram valores de dor em repouso, dor em AVD's, dor em esforço e satisfação, conforme os valores apresentados na Tabela 2.

Tabela 2 - Dados obtidos pela aplicação do questionário PSS - Brasil relacionados a dor e a satisfação

\begin{tabular}{ccccc}
\hline $\begin{array}{c}\text { PACIENTES } \\
\text { REPOUSO }\end{array}$ & $\begin{array}{c}\text { DOR EM } \\
\text { AVD'S }\end{array}$ & $\begin{array}{c}\text { DOR EM } \\
\text { EsFORÇO }\end{array}$ & DOR EM & SATISFAÇÃo \\
\hline S. A & 3 & 7 & 8 & 2 \\
G. B & 3 & 5 & 7 & 4 \\
P. L & 5 & 6 & 9 & 4 \\
M. R. S & 5 & 8 & 10 & 3 \\
C. R. S & 0 & 0 & 3 & 5 \\
A. R. B & 4 & 5 & 5 & 7 \\
P. C. A & 0 & 5 & 10 & 0 \\
A. C.M & 8 & 8 & 10 & 5 \\
C. A.S & 0 & 8 & 0 & 5 \\
H. J.S & 0 & 0 & 10 & 2 \\
N. A.S & 8 & 7 & 8 & 5 \\
E. V.S.S & 5 & 7 & 10 & 5 \\
E. C & 8 & 9 & 10 & 5 \\
D. A. A & 2 & 7 & 4 & 8 \\
N. J. C & 5 & 9 & 10 & 4 \\
Média & 3,73 & 5,53 & 8,13 & 2,19 \\
DesvPad & 2,83 & 3,03 & 2,30 & \\
\hline
\end{tabular}

De acordo com a tabela observou-se que a média de dor em repouso é 3,73\%, a média de dor na realização das AVD's é de 5,53\%, e a dor em esforço obteve a maior média com $8,13 \%$. Pode se verificar também a baixa média de satisfação dos participantes deste estudo atingindo $4 \%$.
Para a função o questionário PSS-Brasil varia de o a 60 pontos sendo o o valor em que o paciente não consegue realizar de forma alguma e quanto mais próximo de 60 melhor o índice de função do ombro, isto é observado da acordo com a Tabela 3. 
Tabela 3 - Porcentagem da pontuação da função do questionário PSS - Brasil

\begin{tabular}{ccc}
\hline PACIENTES & PONTUAÇÃO & $\%$ \\
\hline 1 & 26 & $43,3 \%$ \\
2 & 37 & $61,6 \%$ \\
3 & 29 & $48,3 \%$ \\
4 & 25 & $41,6 \%$ \\
5 & 42 & $70 \%$ \\
6 & 39 & $65 \%$ \\
7 & 1 & $1,6 \%$ \\
8 & 7 & $11,6 \%$ \\
9 & 14 & $23,3 \%$ \\
10 & 46 & $76,6 \%$ \\
11 & 18 & $30 \%$ \\
12 & 6 & $10 \%$ \\
13 & 25 & $41,6 \%$ \\
14 & 36 & $60 \%$ \\
15 & 27 & $45 \%$ \\
\hline
\end{tabular}

Da porcentagem da função de cada paciente foi realizada uma média da porcentagem, sendo que 5 pacientes obtiveram a pontuação de $O$ a $20 \mathrm{com}$ média de 15,33\%, 8 pacientes com pontuação de 20 a $40 \mathrm{com}$ média de $50,80 \%$ e, 2 pacientes de 40 a 60 pontos que obtiveram a média de $73,33 \%$.

Observou-se pela pontuação da função que as atividades as quais os pacientes não conseguem fazer de forma alguma foram: Dormir em cima do braço; Colocar um pote de aproximadamente $5 \mathrm{~kg}$ (saco grande de arroz) em uma prateleira à altura do ombro com o braço afetado esticado; Colocar um pote de aproximadamente $5 \mathrm{~kg}$ (saco grande de arroz) em uma prateleira acima da cabeça com o braço afetado esticado. Conforme demonstra o Gráfico 1.

Gráfico 1 - Relação das atividades as quais os pacientes não conseguem realizar de forma alguma

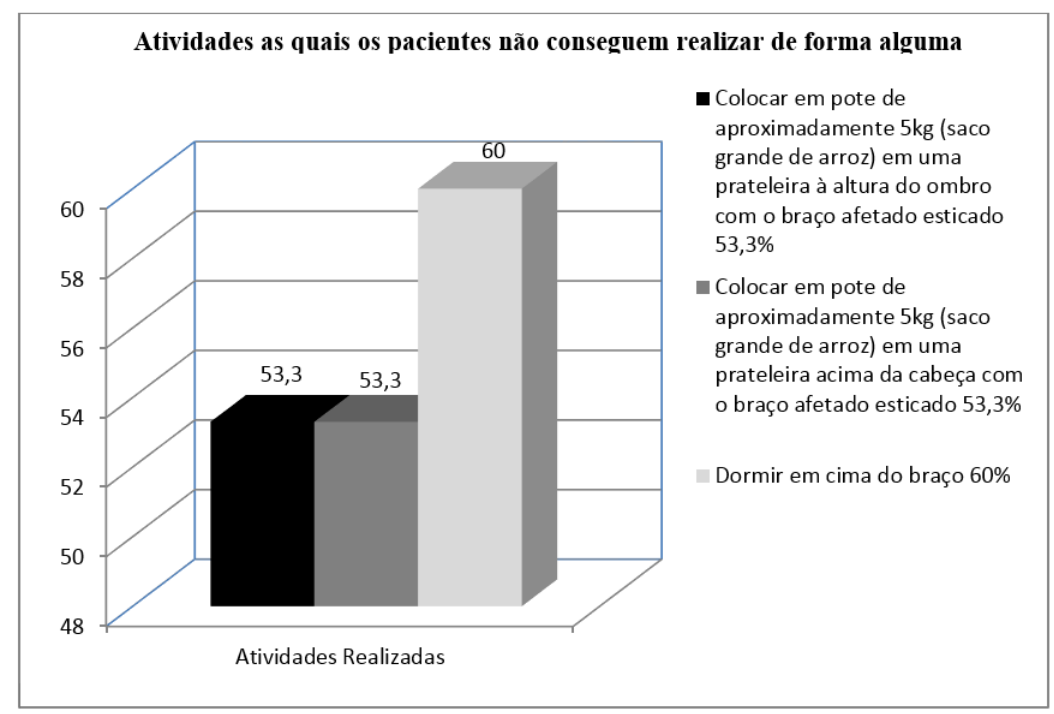


$E$, as atividades que os pacientes realizam sem dificuldade foram: Carregar um livro ou pasta, junto ao corpo, com o braço afetado (40\%); Realizar atividades de higiene pessoal com o braço afetado (33,3\%); Abrir/empurrar a porta com o braço afetado $(33,3 \%)$.
Dos 15 pacientes avaliados com os testes específicos de Neer e Hawkins-kennedy, $100 \%$ apresentaram teste positivo para Hawkins-kennedy e $86,6 \%$ apresentaram teste positivo para Neer, isto pode ser verificado no Gráfico 2.

Gráfico 2 - Valores obtidos pela aplicação dos Testes Específicos: Neer e Hawkins - Kennedy

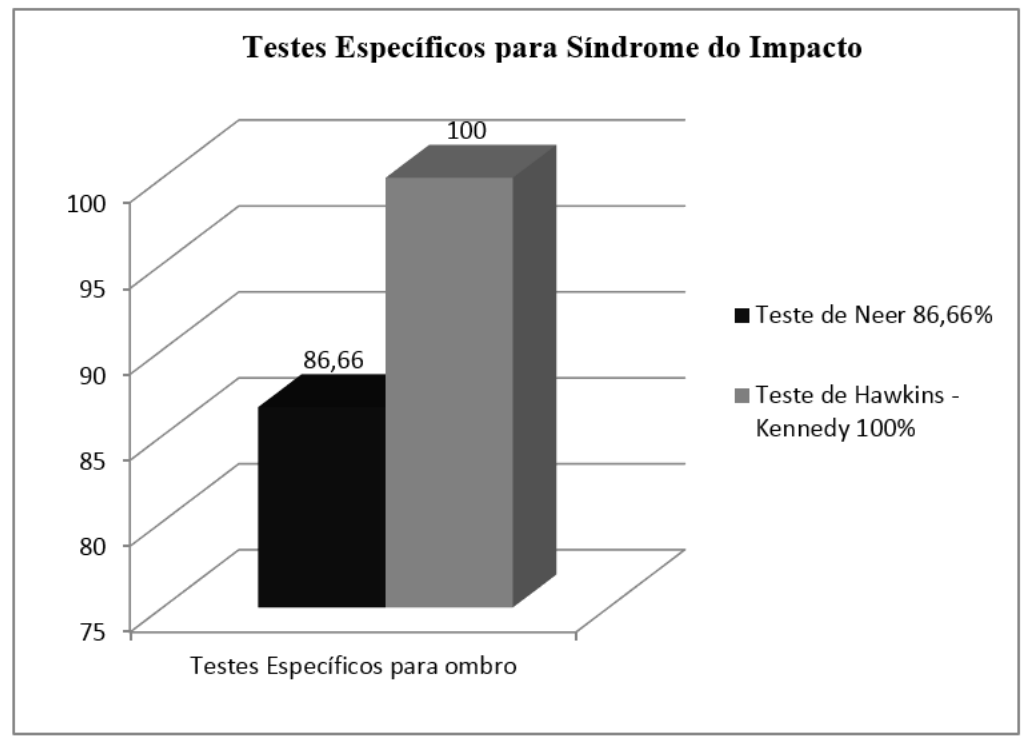

\section{DISCUSSÃO}

A principal sintomatologia da síndrome do impacto é a dor, isso se dá pelo grau de inflamação e pode piorar com os movimentos do ombro e, não pelo tamanho da ruptura. Em todas as fases da lesão isto é presente. Devido ao impacto subacromial ocorre também um arco doloroso de movimento, que acontece durante a elevação do membro superior entre 60 e $120^{\circ} .(6,16,17)$

De um modo geral o déficit dos músculos do manguito rotador é resultado de uma repetitiva tensão que resulta numa perda da função da musculatura para realizar seu papel fisiológico. (3.4) Conforme dados demográficos apresentados após a aplicação do questionário PSS-Brasil, verificou-se que o membro superior direito é o dominante em 12 indivíduos (80\%) e estava acometido em $47 \%$ dos casos, sendo assim o membro superior esquerdo o lado não dominante ele está comprometido em $53 \%$ dos casos. Pode-se verificar nas pesquisas utilizadas para este estudo, que as atividades que requerem mais força e maior sobrecarga, são realizadas com o membro superior não-dominante, e atividades que requerem maior destreza do que força, são realizadas com o membro superior dominante, não gerando tanto impacto sobre o ombro. ${ }^{(18)}$

De acordo com Moreira, $(9)$ indivíduos adultos com idade entre 40 e 50 anos são mais comuns de ocorrer Síndrome do Impacto que é a afecção mais comum na cintura escapular. A média de idade obtida através de achados demográficos dos pacientes foi de 49 anos e 8 meses condizendo com a idade descrita pelo autor acima citado, onde os indivíduos que mais são acometidos pela Síndrome do Impacto tem entre 40 e 50 anos de idade. 
De acordo com a aplicação dos testes irritativos, que indicaram positividade para Síndrome do Impacto, sendo que esta também é gerada uma força sobre o membro superior, mostrou que o resultado dos testes pode ser confirmado pelo aspecto de dor ao esforço, que foi predominante no quesito dor, e baixo grau de satisfação apresentado pelos pacientes durante a aplicação do questionário PSS - Brasil.

A sobrecarga na região do ombro causada pelos movimentos repetitivos ou mau posicionamento do membro superior acarreta dor e incapacidade do ombro afetando aproximadamente $20 \%$ da população em geral. ${ }^{(19)}$

Em geral os pacientes deram-se notas baixas no aspecto de satisfação do ombro. Isto indica ao maior cuidado em que as pessoas têm de se auto -avaliarem. ${ }^{(20,21)}$

A pontuação máxima obtida no quesito função é de 60 pontos indicando o não comprometimento da função do ombro. A pontuação obtida pela maioria dos pacientes variou de o a 40 pontos, mostrando que grande parte dos pacientes com a patologia de síndrome do impacto tem um índice de função baixo, o que corresponde com os testes específicos de Hawkins-Kennedy e Neer. O questionário é realizado com perguntas relacionadas à atividades funcionais que geram maior irritabilidade ao ombro. Sendo assim, a pontuação da função foi baixa porque os pacientes relataram na maioria das atividades não conseguirem realizar de forma alguma, neste caso podemos relacionar com a positividade dos testes que geram grande desconforto na articulação.

De acordo com pontuação da função onde as atividades as quais os pacientes não conseguem fazer de forma alguma foram: Dormir em cima do braço $(60 \%)$; Colocar um pote de aproximadamente $5 \mathrm{~kg}$ (saco grande de arroz) em uma prateleira à altura do ombro com o braço afetado esticado (53,3\%); Colocar um pote de aproximadamente 5 kg (saco grande de arroz) em uma prateleira acima da cabeça com o braço afetado esticado (53,3\%). O que confirma que os movimentos de maior am- plitude são os mais prejudicados e, os que exijam mais carga sobre o ombro ou mau posicionamento do membro.

Silva e Lima ${ }^{(22)}$ descrevem que os movimentos de maior amplitude do ombro que são prejudicados na síndrome, são o de flexão juntamente com o de abdução do ombro gerando a dor, podendo assim influenciar nas atividades de vida diária.

Alguns estudos relatam que quando o braço é usado na posição flexão-rotação interna, a dor, rigidez e frequentemente "fisgadas" são as queixas principais dos pacientes. Dormir em cima do braço afetado, déficits nas AVD's acima da cabeça, são as dificuldades encontradas quando os sintomas acima estão associados. . $^{(3,23)}$

Para o teste específico de Hawkins-kennedy é preciso que a tuberosidade maior do úmero seja direcionada inferiormente ao ligamento coracoacromial e é preciso que o braço seja flexionado a $90^{\circ}$ e realizado uma forçada rotação interna. A dor indica positividade para tendinite do supra-espinhoso porque o tendão é empurrado para a superfície anterior do ligamento coracoacromial e processo coracóide. ${ }^{(24)}$

O teste de Neer é realizado uma elevação rápida do membro superior em rotação interna. Para este autor o teste não é específico, pois o mesmo teste pode indicar capsulite adesiva, instabilidade multidirecional, e, lesões da articulação acromioclavicular. ${ }^{(5)}$

Através da aplicação dos testes específicos para Síndrome do Impacto no ombro, constatou-se que o Teste de Hawkins - Kennedy apresentou maior irritabilidade em comparação ao teste de Neer que se dá pelo posicionamento do membro avaliado e a sobrecarga exercida sobre ele. Pois no teste de Hawkins - Kennedy é realizado uma flexão do ombro a $90^{\circ}$ e uma flexão de cotovelo também a $90^{\circ} \mathrm{e}$ o terapeuta exerce uma rotação interna forçada do ombro, ocasionando maior irritabilidade no músculo supra-espinhoso e, o teste de Neer segundo autor é inespecífico pois avalia também outras patologias. 


\section{CONCLUSÃO}

De acordo com este trabalho o teste de HawkinsKennedy se mostrou mais eficiente para análise da Síndrome do Impacto, por gerar maior irritabilidade ao ombro avaliado.

O questionário é uma ferramenta útil para ser aplicado em pacientes com esta síndrome e que mostrou relação com os testes específicos. Porém, sugerem-se novos estudos que demonstrem parâmetros qualitativos para classificar a função do ombro. Verificou-se que pacientes com Síndrome do Impacto apresentaram dor aos esforços e baixa satisfação da função do ombro.

Os testes específicos de Neer e de Hawkins-Kennedy já são bastante usados na prática fisioterapêutica, e nos dão uma resposta irritativa de dor e consequentemente de funcionalidade do ombro. O questionário PSS- Brasil traz para a avaliação fisioterapêutica uma visão do paciente em relação à dor, a funcionalidade e satisfação do seu ombro. Em comparação com essas duas respostas uma dada pelo paciente e outra através da aplicação dos testes, concluímos que eles são ótimos recursos pois juntos se complementam.

Confirmou-se com este estudo que a associação de testes específicos com a escala PSS, é uma ferramenta útil, tornando desta maneira a avaliação fisioterapêutica mais completa em indivíduos com Síndrome do Impacto.

\section{REFERÊNCIAS}

1. Nordim M, Frankel VH. Biomecânica básica do sistema musculoesquelético. $3^{\text {a }}$. ed. Rio de Janeiro: Guanabara Koogan; 2008.

2. Kendall FP, Mccreary EK, Provance PG, Rodgers MM, Romani WA. Músculos: provas e funções. 5a. ed. Barveri: Manole; 2007.

3. Canavan PK. Reabilitação em medicina esportiva: um guia abrangente. São Paulo: Manole; 2001.
4. Palma MJ de, Johnson EW. Descobrindo e tratando a Síndrome de Impacto do Ombro. 2003, 31.

5. Hebert S, Xavier R. Ortopedia e traumatologia: princípios e prática. $2^{\mathrm{a}}$ ed. Porto Alegre: Artmed; 1998.

6. Andrewa JR, Harrelson GL, Wilk K. Reabilitação física das lesões desportivas. $2^{\mathrm{a}} \mathrm{ed}$. Rio de Janeiro: Guanabara Koogan; 2000.

7. Weinstein SL, Buckwalter JA. Ortopedia de Turek: princípios e sua aplicação. $5^{a}$ ed. São Paulo: Manole; 2000.

8. Michener LA, Mcclure PW, Karduna AR. Anatomical and biomechanical mechanisms of subacromial impingement syndrome. Clin. biomech. 2003;18(5):369-379.

9. Moreira C, Carvalho MAP. Noções práticas de reumatologia. Belo Horizonte: Health; 1998.

1O. Lima GCS, Barbosa EM, Alfiere FM. Análise da funcionalidade e da dor de indivíduos portadores de síndrome do impacto, submetidos à intervenção fisioterapêutica. Fisioter. mov. 2007;20(1):61-69.

11. Beaton DE, Bombardier C, Guillemin F, Ferraz MB. Guidelines for the process of crosscultural adaptation of self-report measures. 2000;25(24):3186-91.

12. Napoles BV, Hoffman CB, Martins J, Oliveira AS. Tradução e adaptação cultural do Penn Shoulder Score para a língua Portuguesa: PSS Brasil. Rev. bras. reumatol. 2010;50(4):389-407.

13. Maage DJ. Avaliação musculoesquelética. $3^{a}$ ed. São Paulo: Manole; $2 \mathrm{OO} 2$.

14. Loudon JK, Bell SL, Johnston JM. Guia clínico de avaliação ortopédica. São Paulo: Manole; 1999.

15. Cipriano JJ. Manual fotográfico de testes ortopédicos e neurológicos. 3a ed. São Paulo: Manole; 1999.

16. Kisner C, Colby LA. Exercícios terapêuticos: fundamentos e técnicas. $3^{a}$ ed. São Paulo: Manole; 1998.

17. Cohen M, Abdalla RJ. Lesões nos esportes. Rio de Janeiro; 2003.

18. Araujo MP, Araujo PP, Caporrino FA, Faloppa F, Albertini WM. Estudo populacional das forças 
das pinça polpa-a-polpa, trípode e lateral. Rev. bras. ortop. 2001;37(11/12):496-504.

19. Teles APS, Almeida NC. Estudo da relação entre a síndrome do impacto do ombro e alterações posturais da cintura escapular. Belém; 2009.

20. Ramos Neto J. Comparação entre duas escalas para diagnóstico auxiliar de joelho e desempenho em testes funcionais [dissertação]. Florianópolis: Universidade do Estado de Santa Catarina; 2000.
21. Pereira Junior AA, Lima WC. Relação entre a função do joelho e índice de massa corporal na Síndrome Patelofemoral. Ter man. 2008;6(26).

22. Silva IA, Lima PP. A Biocinese do Ombro e suas Patologias. 2007. [acesso em: 19 ago. 2011]. Disponível em: http://www.nead.unama.br/site/ bibdigital/pdf/artigos_revistas/87.pdf.

23. Maxey L, Magnusson J. Reabilitação Pós Cirúrgica para Paciente Ortopédico. Rio de Janeiro: Guanabara Koogan; 2003.

24. Metzker CAB. Tratamento conservador na síndrome do impacto no ombro. Fisioter. mov. 2010;23(1):141-151. 